\title{
Photonic bands in two-dimensionally patterned multimode GaN waveguides for light extraction
}

\author{
A. David, ${ }^{\text {a) }}$ C. Meier, R. Sharma, F. S. Diana, S. P. DenBaars, E. Hu, \\ S. Nakamura, and C. Weisbuch ${ }^{\text {b),c) }}$ \\ Materials Department, University of California, Santa Barbara, California 93106 \\ H. Benisty ${ }^{\text {c) }}$ \\ Laboratoire Charles Fabry de l'Institut d'Optique, 91403 Orsay, France
}

(Received 24 November 2004; accepted 14 July 2005; published online 30 August 2005)

\begin{abstract}
We observe experimentally by photoluminescence the band structure and specific emission properties of an in-plane, light-diffracting photonic crystal formed onto a multimode gallium nitride waveguide. Clear-cut two-dimensional photonic crystal effects are reported. Comparison with modeling results in identification of the band structure, provides insight into the light diffraction mechanism and points out design issues for enhancement of the extraction efficiency. (C) 2005 American Institute of Physics. [DOI: 10.1063/1.2039987]
\end{abstract}

Over the last few years, gallium nitride ( $\mathrm{GaN})$-based materials have emerged as a reference blue-green solid-state light source. As for any light emitting diode (LED), the issue of extracting the emitted light has to be dealt with. In a usual LED, $90 \%$ of the light is trapped inside the structure due to the high refractive index of the material, mostly as guided modes. ${ }^{1}$ To improve light extraction, photonic crystals ${ }^{2}$ (PhCs) constitute a promising candidate, besides more classic approaches based on geometrical optics. ${ }^{3}$ Several attempts have been made to incorporate $\mathrm{PhCs}$ as "light extractors," notably for GaN materials. ${ }^{4}$ Recently, electrically injected LED using PhCs have been fabricated, exhibiting overall light emission enhancement of about x 1.5 in the visible range, ${ }^{5}$ and up to $\times 2.5$ in the ultraviolet. ${ }^{6}$ However, detailed understanding of the underlying mechanisms remains open, especially as regards features specific to the $\mathrm{PhCs}$ - as compared to plain one-dimensional (1D) gratings.

In this letter, we present a method for observation of the band structure of a light-extracting photonic crystal in GaN, and bring evidence of its behavior as a true two-dimensional (2D) diffractive element in an overall three-dimensional (3D) structure. We also point out some issues for light extraction.

Two main ways of improving light extraction by means of PhCs can be considered: one is the use of the PhCs band gaps to prevent emission in guided modes; ${ }^{7}$ the other consists in using the $\mathrm{PhC}$ to couple guided modes to radiative modes. ${ }^{8,9}$ The first approach is challenging, due to the need to combine wavelength-sized microcavities with efficient electrical injection and to the effect of nonradiative losses. ${ }^{10}$ Hence, we consider here the second option, namely the use of a $2 \mathrm{D} \mathrm{PhC}$ to turn a guided mode (outside the $\mathrm{PhC}$ ) into a radiative pseudo-guided Bloch mode, lying above the light line of air.

Our structure, designed for photoluminescence studies, is formed as follows: a 2.5 - $\mu$ m-thick GaN buffer layer, a 100 -nm-thick $\mathrm{Al}_{0.3} \mathrm{Ga}_{0.7} \mathrm{~N}$ layer, a 300-nm-thick cap $\mathrm{GaN}$

\footnotetext{
${ }^{a)}$ Also at: Laboratoire Charles Fabry de l'Institut d'Optique, 91403 Orsay, France; electronic mail: davida@engineering.ucsb.edu

b) Also at: Laboratoire de Physique de la Matière Condensée, Ecole Polytechnique, 91128 Palaiseau, France.

${ }^{c)}$ Also at: Genewave, X'Tec, Ecole Polytechnique, 91128 Palaiseau, France.
}

layer which includes a multi-quantum well (QW) region consisting of four-InGaN wells and GaN barriers, and a 40-nmthick $\mathrm{SiO}_{2}$ layer (remaining from the fabrication process). The QWs are grown so as to display broad emission (425$450 \mathrm{~nm}$ at half maximum), in order to characterize a large region of the photonic band structure. The triangular lattice ${ }^{11}$ photonic crystals, defined by electron-beam lithography and dry etching (Fig. 1), consist of rectangular arrays of 160 by $320 \mu \mathrm{m}$, large enough to easily observe light extraction. This geometry could be used in a LED where the emission region is separated from the $\mathrm{PhC}$ extraction region. The following basic $\mathrm{PhC}$ characteristics are thus used: to avoid reflection of the incoming guided mode at the $\mathrm{PhC}$ interface, we try to "match" the vertical profiles of the guided and Bloch modes by using a shallow, perturbative $\mathrm{PhC}$ of $120 \mathrm{~nm}$ depth. ${ }^{12,13}$ The PhC period should be such that the emitted wavelengths fall above the light line, and preferably below the second Bragg order of the $\mathrm{PhC}$ - at higher orders of diffraction, most of the light would be extracted towards the substrate. ${ }^{14,15}$ In our case (modes of effective index neff $\sim 2.45$ ), this corresponds to a PhC period $a=200 \mathrm{~nm}$.

We characterized the PhC structures by angular-resolved photoluminescence spectroscopy. The QW was excited by a $\mathrm{He}-\mathrm{Cd}$ laser, focused $400 \mu \mathrm{m}$ away from the $\mathrm{PhC}$ structure. Luminescence from both the $\mathrm{QW}$ and the $\mathrm{PhC}$ region was collected by a $1 \mathrm{~mm}$ aperture rotating $\left(-90^{\circ}\right.$ to $\left.90^{\circ}\right)$ at a distance of $150 \mathrm{~mm}$ from the sample. The $\mathrm{PhC}$ and laser spot can both be considered located at the center of the rotation movement, considering their proximity at the scale of the

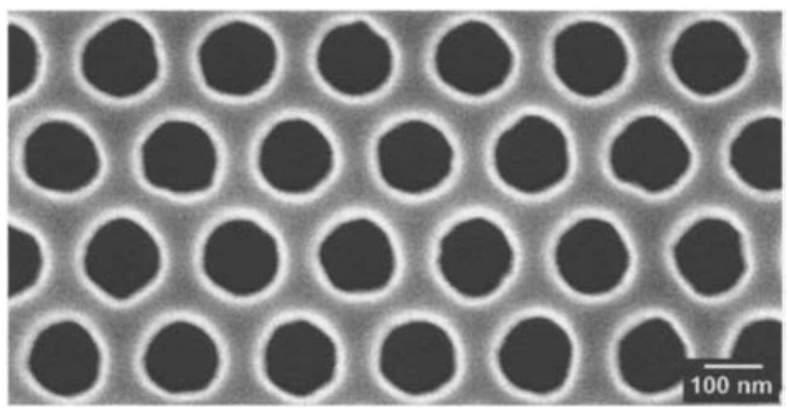

FIG. 1. Scanning electron microscope image of the PhC. 


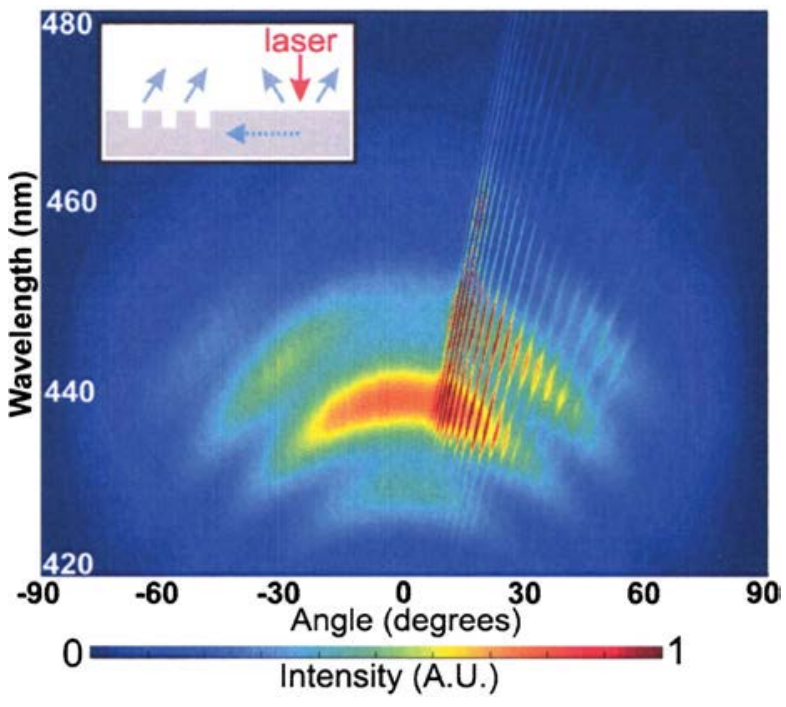

FIG. 2. (Color) Measured angular-resolved spectrum. Inset: Excitation geometry.

setup. Figure 2 shows an angular-resolved spectrum in TE polarization for a $\mathrm{PhC}$ with a filling factor $f=0.3$, excited in the $\Gamma M$ direction. The left hand side of the spectrum is characteristic of an InGaN QW emission in a thick GaN layer: thin film fringes appear, which are dominated by the interference between the light emitted upwards, and the light emitted downwards and reflected at the GaN/sapphire interface-the $\mathrm{SiO}_{2}$ layer weakens the impact of multiple interferences. These fringes modulate the line shape of the QW. On the right hand side of the spectrum, however, additional sharply defined emission lines appear which signal light emission from the $\mathrm{PhC}$ region. Several properties unambiguously confirm that this emission is typical of a $\mathrm{PhC}$ structure. The radiation is strongly directional and occurs backwards, that is, the guided light propagating towards the right of the $\mathrm{PhC}$ region is diffracted in the air to the left. ${ }^{16}$ Indeed, the $\mathrm{PhC}$ period is tuned between the first and second Bragg orders, and the extracted component of the Bloch wave has an in-plane wave vector given by: $\mathbf{k}=\mathbf{k}_{/ /}-\mathbf{G}$, which lies on the left side of the reduced Brillouin zone $\left(\mathbf{k}_{/ /}\right.$ being the in-plane wave vector of the Bloch mode, and $\mathbf{G}$ the reciprocal lattice vector in the $\Gamma M$ direction). The emission is mainly TE polarized (in excess of 80\%). This is expected since most of the light emitted in guided modes is TE and its polarization should be mostly conserved by a shallow $\mathrm{PhC}$. In order to isolate the emission due to the $\mathrm{PhC}$ region, we carefully substract the direct emission of the QW, deduced from the left side of the spectrum. After normalizing this spectrum by the QW line shape, converting the angles into in-plane wave vectors and adding $\mathbf{G}$, we obtain the dispersion relation of the PhC bands [Fig. 3(a)], folded in the first Brillouin zone.

We notice that two sets of bands are present: the first (set A) are intense lines with a strong slope, while the second (set B) are weaker and with a lower slope. Anticrossings appear between the two sets of bands. These band sets can be understood simply from the band structure of an infinite 2D photonic crystal with small photonic strength [Fig. 4(a)]. In the considered region, two Bloch modes exist in the infinite 2D photonic crystal. Mode $A$ has properties close to a plane wave (nearly free photon dispersion folded by periodicity), while mode $B$ (a nearly degenerate doublet) is a more comDownloaded 31 Aug 2005 to 129.104.38.4. Redistribution subject

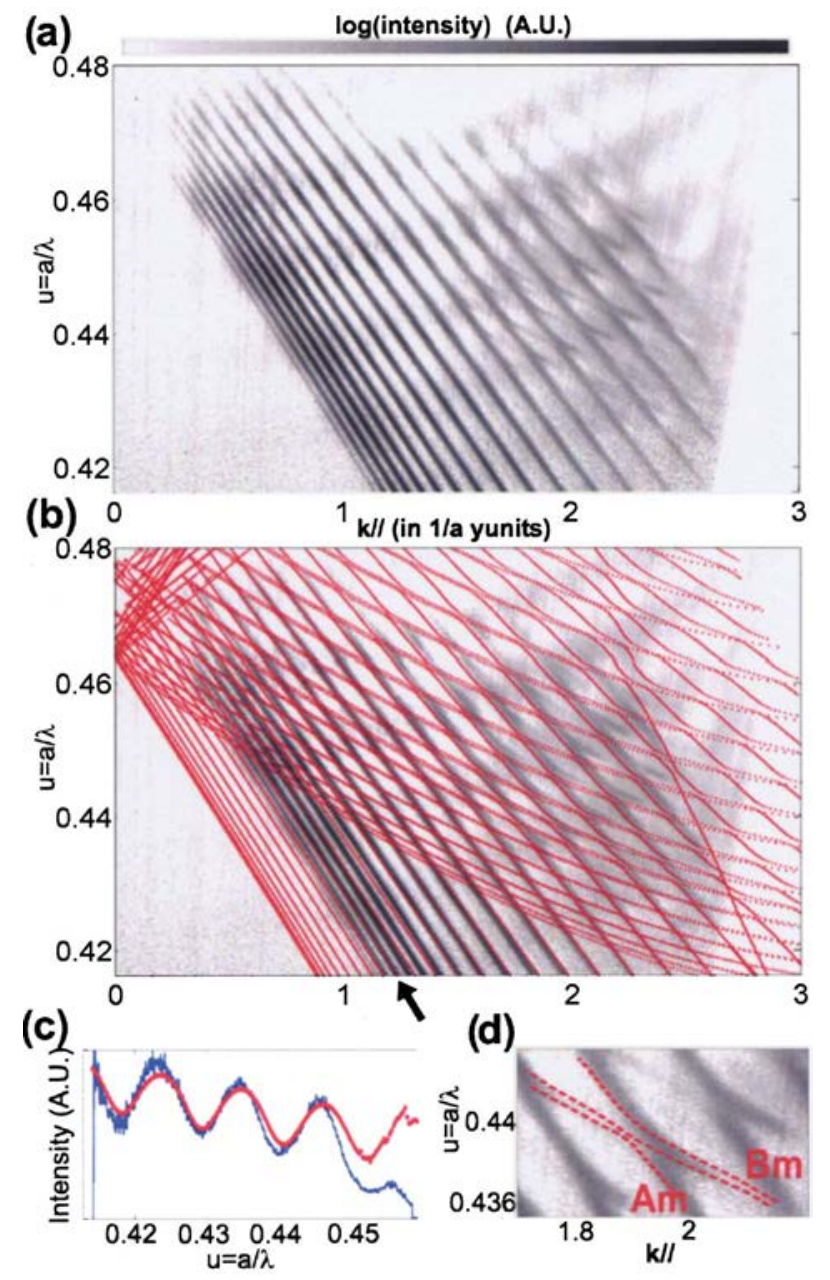

FIG. 3. (Color) (a) Measured band structure of the PhC (log scale). (b) Measured and theoretical (red dots) band structure. (c) Measured (blue) and theoretical (red) emission along a band [indicated by an arrow on figure (b)]. (d) Zoom on an anticrossing.

plex Bloch mode, essentially a superposition of two plane wave harmonics. In the real structure, which is multimode in the vertical direction, each of the vertical modes $(m=1,2 \ldots)$ has its own effective index $n_{m}$, and gives rise to two bands $A_{m}$ and $B_{m}$ [Fig. 4(b)]. These two sets correspond to those of the measured band structure.

To check quantitatively this interpretation and account for the radiation losses, we modeled the full 3D structure with a spectral-domain rigorous electromagnetic solver, ${ }^{17}$ which provides both the dispersion and extraction rates of the Bloch modes. GaN refractive index values were taken

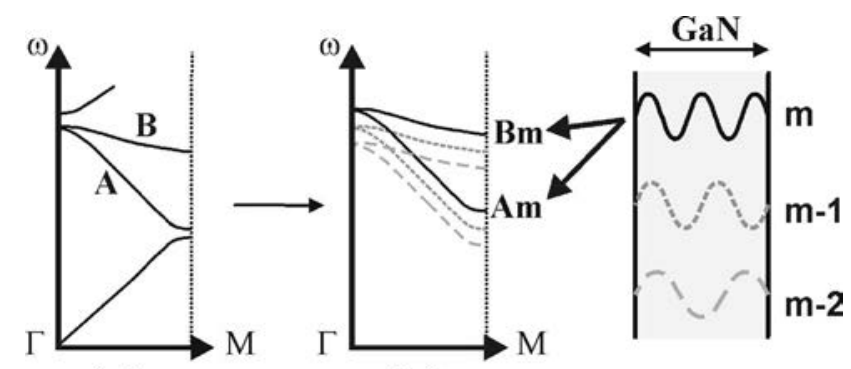

(a)

(b)

FIG. 4. (a) Two-dimensional band structure of a photonic crystal in the $\Gamma M$ direction. (b) Corresponding band structure in a multimode waveguide: mode $m$ gives rise to two $\mathrm{PhC}$ bands, $A_{m}$ and $B_{m}$. 
from Ref. 18. The general dispersion obtained [Fig. 3(b)]using no fitting parameter-is in very good agreement with the measurement, including bands position and anticrossings. Several of the calculated bands are not observed experimentally: we checked that they correspond to the lowest-order guided modes $(m \leqslant 7)$. Their overlap with the photonic crystal region is lowest, and their extraction rate is very poor. This should be taken into account for optimal design of the overall layers and $\mathrm{PhC}$ structure.

The observation of anticrossings with B-type modes is especially significant since, unlike pure A-type modes, those are specific to 2D photonic crystals. More precisely, if the $\mathrm{PhC}$ merely behaved as a superposition of independent 1D gratings, only unperturbed A-type modes would be observed. ${ }^{19}$ Besides, owing to the nontrivial Bloch mode structure of B-type modes (superposition of two waves), it is interesting to observe in practice that an incoming guided mode can excite Bloch modes at the anticrossings-even though the coupling is not strong, as can be seen from their relatively weak intensity compared to pure A-type modes. Anticrossing between some of the $A_{m}$ and $B_{n}$ modes $(m$ $\neq n$ ) is caused by the coupling due to refractive index modulation. Therefore, anticrossing is mainly observed (both theoretically and experimentally) for those modes which strongly overlap with the PhC holes [Fig. 3(d)].

It is also interesting that the measured emission intensity collapses at high frequencies $(u>0.47$, e.g., $\lambda<425 \mathrm{~nm})$, that is, when the wave vector is close to the Brillouin zone center, whereas calculation shows that Bloch modes with good extraction rate still exist at that frequency. This appears on Fig. 3(c) where the emission of a given $A_{m}$ band is displayed (oscillations stem from thin film interferences, as above). This discrepancy may partly stem from poor coupling of the guided mode to Bloch modes close to the band gap, where the structure of Bloch modes is significantly altered by the PhC effect-even for shallow PhCs. It would be easy to circumvent this by designing the photonic crystal to operate far enough from a Bragg order. However, this region of the band structure also corresponds to the low-wavelength tail of the QW emission, where reabsorption of guided modes by the QW is strongest. The relative effect of mode coupling and absorption still calls for clarification. This should be compared to the case of Ref. 5, where light is created directly in the photonic crystal region, and coupling and absorption are plausibly not issues: extraction is observed at the third Bragg order (nearly vertical angles). However, current injection then becomes an issue because of the presence of the photonic crystals above the active region.

The experiments were also carried out for filling factors 0.2 and 0.37 , and for $\mathrm{PhCs}$ oriented in the $\Gamma K$ direction, yielding results supporting the above analysis.

Finally, separate microphotoluminescence measurements enable us to measure a band-averaged attenuation rate for light propagating in the $\mathrm{PhC}$ structure. Upon varying the collection point along the $\mathrm{PhC}$ area, we observe an exponential decay of the extracted radiation. We obtain a value of $L_{\text {decay }} \sim 100 \mu \mathrm{m}$, somehow independent of the wavelength, in good agreement with the values due to radiation loss obtained from simulation $(80-140 \mu \mathrm{m}$, depending on the band considered). This points out that, in order to extract most of the guided light, the photonic crystal should spread quite far around the injected region $(\sim 200 \mu \mathrm{m}$ in our case).

In conclusion, we have characterized experimentally the band structure and emission properties of a light-diffracting 2D PhC defined in a GaN structure. We provided a theoretical interpretation based on a 3D spectral model. Measurements reveal peculiar features which are specific of a $2 \mathrm{D}$ periodic structure. Modeling of the structure shows satisfying agreement with the measurement, and raises several design issues for light extraction: existence of unextracted modes, desirable size of the $\mathrm{PhC}$, possible poor coupling for frequencies close to the second Bragg order. This clearly calls for further optimization, fully considering both $\mathrm{PhC}$ and vertical heterostructure parameters, and also leaves good prospects for light extraction in multimode structures.

${ }^{1}$ H. Benisty, H. De Neve, and C. Weisbuch, IEEE J. Quantum Electron. 34, 1612 (1998); 34, 1632 (1998).

${ }^{2}$ E. Yablonovitch, Phys. Rev. Lett. 58, 2059 (1987).

${ }^{3}$ M. R. Krames, M. Ochiai-Holcomb, G. E. Holfer, C. Carter-Coman, E. I. Chen, I. H. Tan, P. Grillot, N. F. Gardner, H. C. Chui, J. W. Huang, S. A. Stockman, F. A. Kish, M. G. Craford, S. T. Tan, C. P. Kocot, M. Hueschen, J. Posselt, B. Loh, G. Sasser, and D. Collins, Appl. Phys. Lett. 75, 2365 (1999).

${ }^{4}$ T. N. Oder, J. Shakya, J. Y. Lin, and H. X. Jiang, Appl. Phys. Lett. 83, 1231 (2003).

${ }^{5}$ J. J. Wierer, M. R. Krames, J. E. Epler, N. F. Gardner, M. G. Craford, J. R. Wendt, J. A. Simmons, and M. M. Sigalas, Appl. Phys. Lett. 84, 3885 (2004).

${ }^{6}$ J. Shakya, K. H. Kim, J. Y. Lin, and H. X. Jiang, Appl. Phys. Lett. 85, 142 (2004).

${ }^{7}$ S. Fan, P. R. Villeneuve, J. D. Joannopoulos, and E. F. Schubert, Phys. Rev. Lett. 78, 3294 (1997).

${ }^{8}$ M. Rattier, H. Benisty, R. P. Stanley, J.-F. Carlin, R. Houdré, U. Oesterle, C. J. M. Smith, C. Weisbuch, and T. F. Krauss, IEEE J. Sel. Top. Quantum Electron. 8, 238 (2002).

${ }^{9}$ H. Rigneault, F. Lemarchand, and A. Sentenac, J. Opt. Soc. Am. A 17, 1048 (2000).

${ }^{10}$ R. K. Lee, Y. Xu, and A. Yariv, J. Opt. Soc. Am. B 17, 1438 (2000).

${ }^{11}$ This choice is due to the sixfold symmetry of the triangular lattice, which should display quite isotropic behavior and constitute a first step towards omnidirectional extraction. Further study of the directionality issue will be reported elsewhere.

${ }^{12}$ M. Palamaru, and Ph. Lalanne, Appl. Phys. Lett. 78, 1466 (2001).

${ }^{13}$ S. G. Johnson, P. Bienstman, M. A. Skorobogatiy, M. Ibanescu, E. Lidorikis, and J. D. Joannopoulos, Phys. Rev. E 66, 06608 (2002).

${ }^{14}$ M. Rattier, H. Benisty, E. Schwoob, C. Weisbuch, T. F. Krauss, C. J. M. Smith, R. Houdre, and U. Oesterle, Appl. Phys. Lett. 83, 1283 (2003).

${ }^{15}$ M. Hagberg, N. Erikkson, and A. Larsson, IEEE J. Quantum Electron. 32, 1596 (1996).

${ }^{16}$ More precisely, the light is diffracted in the left half space, with an azimuthal direction depending on the (small) incident angle of the guided mode with respect to the $\Gamma M$ direction. Angular measurements slightly off the $\Gamma M$ direction reveal similar results as those reported.

${ }^{17}$ In our method, designed to account for losses by diffraction, the electric field is decomposed in a plane wave basis along the periodic directions of the $\mathrm{PhC}$, and discretized in real space in the vertical direction. The frequency of light is imposed, and Maxwell's equations take the form of an eigenvalue problem where we solve for the wave vector and Bloch mode profile. Details will be published elsewhere.

${ }^{18}$ H. Y. Zhang, X. H. He, Y. H. Shih, M. Schurman, Z. C. Feng, and R. A. Stall, Opt. Lett. 21, 1529 (1996).

${ }^{19}$ Due to the directionality of the incoming guided modes, B-modes would not be excited as they stem from guided modes propagating off the $Г \mathrm{M}$ direction. 